\title{
Quantum interference in an electron-hole graphene ring system
}

\author{
D. Smirnov, H. Schmidt and R. J. Haug \\ Institut für Festkörperphysik, Leibniz Universität Hannover, Appelstr. 230167 Hannover, Germany
}

\begin{abstract}
Quantum interference is observed in a graphene ring system via the Aharonov Bohm effect. As graphene is a gapless semiconductor, this geometry allows to study the unique situation of quantum interference between electrons and holes in addition to the unipolar quantum interference. The period and amplitude of the observed Aharonov-Bohm oscillations are independent of the sign of the applied gate voltage showing the equivalence between unipolar and dipolar interference.
\end{abstract}

Keywords: Aharonov-Bohm effect, graphene, quantum interference phenomena, semiconductor materials

The recent discovery of graphene [1] offers great potential in fundamental research of electron transport and in electronic devices due to the specific energy dispersion of such monolayers of graphite [2]. One of the remarkable effects of the bandstructure in graphene is that both electron and holes can be induced in one sample controlled with local gates [3, 4]. For such experiments, the Klein tunneling was predicted [5]. Also the interference of charge carriers due to the Aharonov-Bohm (AB) effect [6] was heavily discussed recently for graphene monolayers, but only few experimental works have been carried out $[7,8]$.

The sample was fabricated via a standard procedure: the graphene flake was produced by mechanical exfoliation and deposited on a $285 \mathrm{~nm}$ thin layer of $\mathrm{SiO}_{2}$ on top of a heavily p-doped silicon wafer, which was used as a backgate (BG) during the measurements. Electron beam lithography and oxygen plasma etching were used to define a ring with an inner radius of $220 \mathrm{~nm}$ and an outer radius of $360 \mathrm{~nm}$ (Fig. 1(d)). In a second step Chromium/Gold contacts were evaporated. In the third step another layer of Polymethylmethacrylate (PMMA) was deposited on top of the flake to enable the fabrication of a topgate (TG) using the PMMA as an insulator $[9,10]$. The Chromium/Gold topgate was evaporated over one arm of the ring. All shown measurements are four probe measurements and have been performed in a $\mathrm{He}^{3}$ - cryostat with a base temperature of $500 \mathrm{mK}$. The characterization of the sample as monolayer graphene were done via magnetotransport measurements (see [11]).

Figure 1 shows measurements over the ring versus the backgate voltage. The magnetic field was swept in a limited range around zero magnetic field in order to avoid the occurrence of Shubnikov-de Haas oscillations. Figure 1(b) shows the ring resistance as a function of magnetic field. Oscillations are seen over the whole magnetic-field range. These oscillations have a maxi- mum visibility of $1 \%$ and can be identified as $\mathrm{AB}$ oscillations. Figure 1 shows the $\mathrm{AB}$ oscillations for electrons (b) and holes (c) with a subtracted background obtained over a running average with a minimum of one period $[7,8,11]$. The measurements are taken for comparable charge carrier concentrations $\left(U_{B G}=34 \mathrm{~V}\right.$ for electrons and $U_{B G}=-16 \mathrm{~V}$ for holes). Whereas there is no clear observation of oscillations at the charge neutrality point (CNP) oscillations are detected away from CNP. However we do not observe higher amplitudes with rising carrier concentration for electrons nor for holes.

Figure 1(e) shows the Fourier spectrum of the oscillations presented in Fig. 1(b). The Fourier spectrum has a peak at $\Delta B^{-1}=62 \mathrm{~T}^{-1}$ which corresponds to a period of $\Delta B_{A B}=16 \mathrm{mT}$. The expected period for a ring $\Delta B_{A B}=h /\left(e \pi r^{2}\right)$ with an average radius $r$ of $290 \mathrm{~nm}$ is
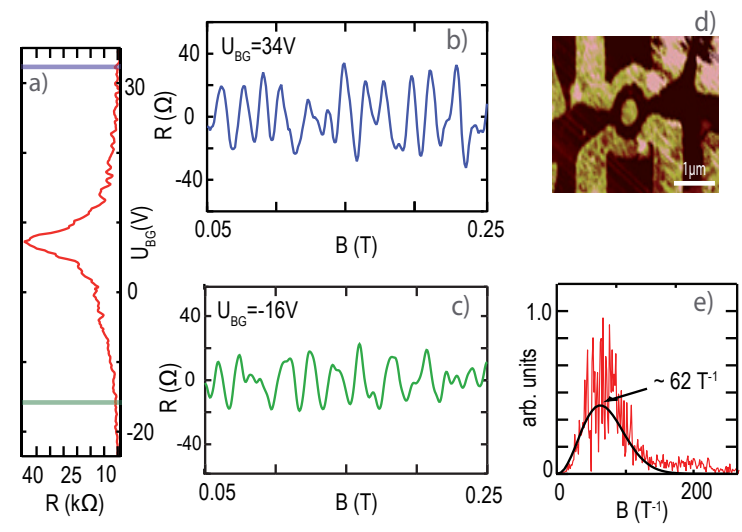

FIGURE 1. (a) Four probe measurements over the ring versus backgate voltage. $\mathrm{AB}$ oscillations for holes (b) and electrons (c) with comparable carrier concentration. (d) The Atomic Force Microscope picture of the ring. (e) Fourier spectrum of the $\mathrm{AB}$ oscillations with a Gaussian fit. 
$15.6 \mathrm{mT}$, so the measured oscillations fit the size of the ring. The black curve is a Gaussian fitted to the Fourier spectrum. The curve illustrates the period of the oscillations and the spreading which can be a hint towards the different paths possible within the geometric width of the ring. In the Fourier spectrum we observe a tail around $\Delta B^{-1}=125 \mathrm{mT}^{-1}$, which can be an indication towards the second harmonic and explains the strong modulation of the oscillations. The phase coherence length of our system has to be shorter than two times the ring circumference, since there appear only indications of a second harmonic.

In contrast to the previous $\mathrm{AB}$ experiments [7, 8], our experimental set-up (see Fig. 2(d)) allows for the generation of different charge carriers in the two arms of the ring by varying the backgate and the topgate voltage. So AB oscillations with different charge carrier concentration inside the ring can be performed. One of these measurement is presented in Fig. 2. It shows the resistance measurements over the ring with a fixed backgate voltage, so that the leads and one side of the ring have holes as charge carriers with a concentration of $p=8.6 \cdot 10^{15} \mathrm{~m}^{-2}$ due to the fixed backgate voltage, whereas the other side of the ring has a different charge concentration due to the influence of the topgate voltage.

Two sets of oscillations with a subtracted background are shown in Fig. 2(c) (unipolar) and 2(e) (dipolar). The charge carrier concentrations used in these two measurements are of similar magnitude but different polarity. In both situations the absolute amplitude is quite similar.
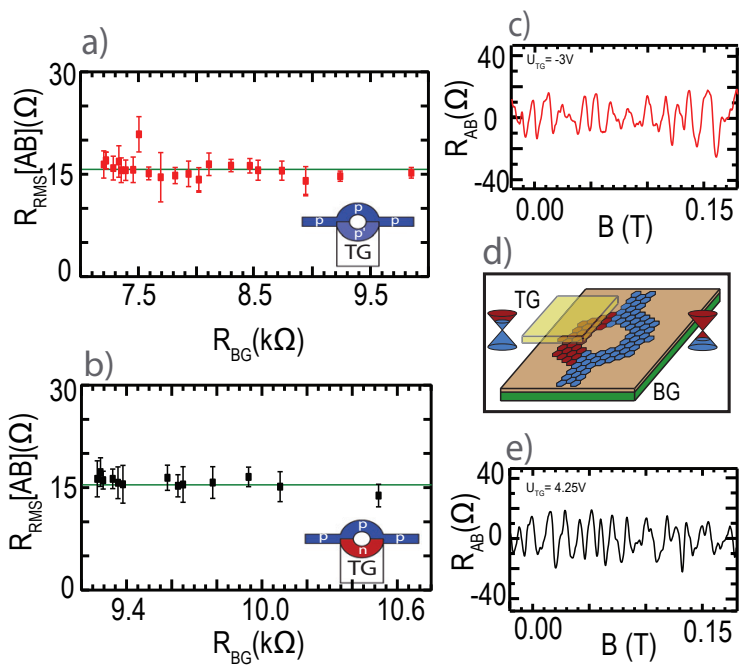

FIGURE 2. The effective $\mathrm{AB}$ oscillations amplitude (RMS) versus the background resistance for a unipolar (a) and bipolar (b) system. $\mathrm{AB}$ oscillations with a subtracted background for only holes (c) and electrons and holes (e). The schematic picture of the topgate position over one arm of the ring (d).
The period of the oscillations is also not affected by the unipolar or dipolar situation. The $\mathrm{AB}$ oscillations were analyzed for a number of different topgate voltages. The RMS values of the absolute amplitude is analyzed as described before and is constant with an average value of $16.1 \pm 3.9 \Omega$. It does not change for different charge carrier type and concentrations.

In a previous work with a global backgate a linear dependance for the amplitude versus the background conductance was found [7]. In Fig. 2 the effective amplitude in our sample is shown versus the background resistance for unipolar (a) and bipolar (b) interference. Such a change in the background resistance was induced by sweeping the local topgate voltage. The amplitude stays constant in the range of error bars and is not effected by the background resistance. This behavior does not depend on the polarity and is the same for unipolar and bipolar situation. However the change of the background resistance through the topgate voltage is relatively small in comparison to the one induced by the backgate voltage because of the small area that is covered by the topgate.

In conclusion we have reported $\mathrm{AB}$ oscillations in a monolayer graphene ring system. Our measurements show that $\mathrm{AB}$ oscillations are possible in an electron-hole system and the period and amplitude of the bipolar oscillations are independent of the background resistance.

\section{REFERENCES}

1. K. S. Novesolelov, A. K. Geim, S. V. Morozov, D. Jiang, Y. Zhang, S. V. Dubonos, I. V. Grigorieva, and A. A. Firsov, Science 306, 666, 2004.

2. P. R. Wallace, Phys. Rev. 71, 622-634, 1947.

3. B. Özyilmaz, P. Jarillo-Herrero, D. Efetov, D. A. Abanin, L. S. Levitov, and P. Kim, Phys. Rev. Lett. 99, 166804 , 2007.

4. G. Liu, J. Velasco Jr., W. Bao, and C. N. Lau, Appl. Phys. Lett. 92, 203103, 2008.

5. M. I. Katsnelson, K. S. Novoselov and A. K. Geim, Nat Phys 2, 620, 2006.

6. Y. Aharonov and D. Bohm, Phys. Rev. 115, 485, 1959.

7. S. Russo, J. B. Oostinga, D. Wehenkel, H. B. Heersche, S. S. Sobhani, L. M. K. Vandersypen, and A. F. Morpurgo, Phys. Rev. B 77, 085413, 2008.

8. M. Huefner, F. Molitor, A. Jacobsen, A. Pioda, C. Stampfer, K. Ensslin, and T. Ihn, New Journal of Physic 12, 043054, 2010.

9. B. Huard, J. A.Sulpizio, N. A.Stander, K. Todd, B.Yang, and D. Goldhaber-Gordon, Phys. Rev. Lett. 98, 236803, 2007.

10. H. Schmidt, T. Lüdtke, P. Barthold, E. McCann, V. I. Fal'ko, and R. J. Haug, Appl. Phys. Lett. 93, 172108, 2008.

11. D. Smirnov, H. Schmidt, and R. J. Haug, Appl. Phys. Lett. 100, 203114, 2012. 
AIP Conference Proceedings is copyrighted by AIP Publishing LLC (AIP). Reuse of AIP content is subject to the terms at: http://scitation.aip.org/termsconditions. For more information, see http://publishing.aip.org/authors/rights-and-permissions. 